ELECTRONIC RESEARCH ANNOUNCEMENTS OF THE AMERICAN MATHEMATICAL SOCIETY

Volume 11, Pages 71-77 (September 23, 2005)

S $1079-6762(05) 00149-6$

\title{
LOWER BOUNDS FOR THE SPECTRAL FUNCTION AND FOR THE REMAINDER IN LOCAL WEYL'S LAW ON MANIFOLDS
}

\author{
DMITRY JAKOBSON AND IOSIF POLTEROVICH
}

(Communicated by Svetlana Katok)

\begin{abstract}
We announce asymptotic lower bounds for the spectral function of the Laplacian and for the remainder in the local Weyl's law on Riemannian manifolds. In the negatively curved case, methods of thermodynamic formalism are applied to improve the estimates. Our results develop and extend the unpublished thesis of A. Karnaukh. We discuss some ideas of the proofs; for complete proofs see our extended paper on the subject.
\end{abstract}

\section{Spectral function and the Weyl's law}

Let $X$ be a compact Riemannian manifold of dimension $n \geq 2$ with the metric $\left\{g_{i j}\right\}$ and the volume $V$. Let $\Delta$ be the Laplacian on $X$ with eigenvalues $0=\lambda_{0}<$ $\lambda_{1} \leq \lambda_{2} \leq \cdots$ and the corresponding orthonormal basis $\left\{\phi_{i}\right\}$ of eigenfunctions: $\Delta \phi_{i}=\lambda_{i} \phi_{i}$.

Given $x, y \in X$, let

$$
N_{x, y}(\lambda)=\sum_{\sqrt{\lambda_{i}} \leq \lambda} \phi_{i}(x) \phi_{i}(y)
$$

be the spectral function of the Laplacian. On the diagonal $x=y$ we denote it simply $N_{x}(\lambda)$. If $N(\lambda)=\#\left\{\sqrt{\lambda_{i}} \leq \lambda\right\}$ is the eigenvalue counting function, then $N(\lambda)=\int_{X} N_{x}(\lambda) d V$. Let

$$
\sigma_{n}=\frac{2 \pi^{n / 2}}{n \Gamma(n / 2)}
$$

be the volume of the unit ball in $\mathbf{R}^{n}$. The asymptotic behavior of the spectral and the counting functions is given by ([13]; see also [28]):

$$
\begin{aligned}
N_{x, y}(\lambda) & =O\left(\lambda^{n-1}\right), \quad x \neq y ; \\
N_{x}(\lambda) & =\frac{\sigma_{n}}{(2 \pi)^{n}} \lambda^{n}+R_{x}(\lambda), \quad R_{x}(\lambda)=O\left(\lambda^{n-1}\right) ; \\
N(\lambda) & =\frac{V \sigma_{n}}{(2 \pi)^{n}} \lambda^{n}+R(\lambda), \quad R(\lambda)=O\left(\lambda^{n-1}\right) .
\end{aligned}
$$

Received by the editors June 7, 2005.

2000 Mathematics Subject Classification. Primary 58J50; Secondary 35P20, 37C30, 81Q50.

Key words and phrases. Weyl's law, spectral function, wave kernel, negative curvature, Anosov flow, thermodynamic formalism.

The first author was supported by NSERC, FQRNT, Alfred P. Sloan Foundation fellowship and Dawson fellowship. The second author was supported by NSERC and FQRNT.

(C)2005 American Mathematical Society Reverts to public domain 28 years from publication 
We refer to the asymptotics of $N_{x}(\lambda)$ as the local Weyl's law, the asymptotics for $N(\lambda)$ being the usual Weyl's law for the distribution of eigenvalues. The upper bounds for $R(\lambda)$ and $R_{x}(\lambda)$ are attained for round spheres and hence are sharp. Both local and integrated remainder estimates for the Weyl's law on manifolds under various geometric conditions have been actively studied in the last forty years (see [2], 8], 10], 14], 21], 24], 26], 29], 31], etc).

In the present paper we focus on asymptotic lower bounds for the spectral function and for the remainder in local Weyl's law.

We recall that $f_{1}(\lambda)=\Omega\left(f_{2}(\lambda)\right)$ for an arbitrary function $f_{1}$ and a positive function $f_{2}$ means $\lim \sup _{\lambda \rightarrow \infty}\left|f_{1}(\lambda)\right| / f_{2}(\lambda)>0$.

Theorem 1.3. Let $X$ be a compact n-dimensional Riemannian manifold, and let $x, y \in X$ be two points that are not conjugate along any shortest geodesic joining them. Then

$$
N_{x, y}(\lambda)=\Omega\left(\lambda^{\frac{n-1}{2}}\right) .
$$

Let us now formulate the on-diagonal counterpart of Theorem 1.3. Consider the heat trace asymptotics as $t \rightarrow 0^{+}$:

$$
\sum_{i} e^{-\lambda_{i} t} \sim \frac{1}{(4 \pi t)^{n / 2}} \sum_{j=0}^{\infty}\left(\int_{X} a_{j}(x) d \mathrm{vol}\right) t^{n},
$$

where $a_{j}(x)$ are the local heat invariants of $X$. Let $\kappa_{x}=\min \left\{j \geq 1 \mid a_{j}(x) \neq 0\right\}$. If $a_{j}(x)=0$ for all $j \geq 1$, we set $\kappa_{x}=\infty$. We recall that $a_{1}(x)=\frac{\tau(x)}{6}$, where $\tau(x)$ is the scalar curvature of $X$ at the point $x$.

Theorem 1.6. Let $X$ be an $n$-dimensional Riemannian manifold and $x \in X$ an arbitrary point. If $n-2 \kappa_{x}-1>0$, then

$$
R_{x}(\lambda)=\Omega\left(\lambda^{n-2 \kappa_{x}-1}\right) .
$$

If $X$ has no conjugate points, then

$$
R_{x}(\lambda)=\Omega\left(\lambda^{\frac{n-1}{2}}\right) .
$$

Remark 1.9. If $n-4 \kappa_{x}-1<0$, then the bound (1.8) is better than the bound (1.7). If the scalar curvature $\tau(x) \neq 0$, then (1.7) becomes $R_{x}(\lambda)=\Omega\left(\lambda^{n-3}\right)$.

Estimate (1.8) should be compared with the Hardy-Landau lower bound for the remainder in the Gauss circle problem or, equivalently, for the remainder in the Weyl's law on a 2-dimensional flat square torus. We note that (1.8) gives the same exponent as in the Hardy-Landau bound for any surface without conjugate points. In dimension 3 the exponent in (1.8) is also consistent with the lower bound due to Szegö for the error term in the sphere problem (see [30]). However, for a similar counting problem in a ball of dimension $n \geq 4$, the sharp error estimate is $\Omega\left(\lambda^{n-2}\right)$ which is larger than (1.8).

\section{Estimates FOR NEGATIVELY CURVED MANIFOLDS}

Asymptotic lower bounds (1.4), (1.7), and (1.8) can be improved for manifolds of negative curvature. We assume that for any pair of directions $\xi, \eta$ the sectional curvature $K(\xi, \eta)$ satisfies

$$
-K_{1}^{2} \leq K(\xi, \eta) \leq-K_{2}^{2}
$$


Apart from the standard wave equation techniques (cf. [10, [2], 16]) our method uses thermodynamic formalism (see, for example, 6], 20]). Let $M$ be the universal cover of $X$. Let $G^{t}$ be the geodesic flow on the unit tangent bundle $S M$ and let $E_{\xi}^{u}$ be the unstable subspace for $G^{t}, \xi \in S M$. The Sinai-Ruelle-Bowen potential is a Hölder continuous function $\mathcal{H}: S M \rightarrow \mathbf{R}$ which for any $\xi \in S M$ is defined by the formula (see [7], 27])

$$
\mathcal{H}(\xi)=\left.\left.\frac{d}{d t}\right|_{t=0} \ln \operatorname{det} d G^{t}\right|_{E_{\xi}^{u}}
$$

For any continuous function $f: S M \rightarrow \mathbf{R}$ one can define the topological pressure

$$
P(f)=\sup _{\mu}\left(h_{\mu}+\int f d \mu\right),
$$

where the supremum is taken over all $G^{t}$-invariant measures $\mu$, and $h_{\mu}$ denotes the measure-theoretic entropy of the geodesic flow (see [6]). In particular $P(0)=h$, where $h$ is the topological entropy of the flow.

Theorem 2.4. The remainder in the local Weyl's law on an $n$-dimensional compact negatively curved manifold satisfies

$$
R_{x}(\lambda)=\left\{\begin{array}{l}
\Omega\left(\lambda^{\frac{n-1}{2}}(\log \lambda)^{\frac{P(-\mathcal{H} / 2)}{h}-\delta}\right) \quad \forall \delta>0, \quad n \leq 5 ; \\
\Omega\left(\lambda^{n-3}\right), \quad n \geq 6 .
\end{array}\right.
$$

One can show that the $\frac{P(-\mathcal{H} / 2)}{h}$ is estimated in terms of curvatures as follows:

$$
\frac{P(-\mathcal{H} / 2)}{h} \geq \frac{K_{2}}{2 K_{1}}>0 \text {. }
$$

We note that in dimensions $n \leq 5$ the main contribution to the remainder comes from the oscillating terms corresponding to geodesic loops. At the same time, in dimensions $n \geq 6$ the contribution of the singularity at $t=0$ dominates the remainder. This explains different bounds in Theorem 2.4 for low and high dimensions.

Away from the diagonal, we get

Theorem 2.6. On a compact $n$-dimensional negatively curved manifold the spectral function $N_{x, y}(\lambda)$ satisfies, for any $\delta>0$ and $x \neq y$,

$$
N_{x, y}(\lambda)=\Omega\left(\lambda^{\frac{n-1}{2}}(\log \lambda)^{\frac{P(-\mathcal{H} / 2)}{h}-\delta}\right) .
$$

2.1. Discussion: remainder estimates on negatively curved surfaces. Let us compare the results of this section with some known facts and conjectures regarding the error term in Weyl's law on negatively curved surfaces.

A lower bound for the local remainder $R_{x}(\lambda)$ on negatively curved surfaces was proved in an unpublished Princeton Ph.D. thesis [16]. On surfaces of constant negative curvature (2.5) coincides with its analogue in [16, but the techniques of thermodynamic formalism for hyperbolic flows allow us to improve the results of [16] when the curvature is variable. We do not need the hypothesis $K_{1} / K_{2}<2$ of [16] to get a logarithmic improvement in the estimates, and moreover, we get higher powers of the logarithm.

For surfaces of constant negative curvature it is proved in 23 that $R(\lambda)=$ $\Omega\left((\log \lambda)^{\frac{1}{2}-\delta}\right)$ for any $\delta>0$. In a work in progress we aim to generalize this bound 
for surfaces of variable negative curvature using the methods of thermodynamic formalism announced in the present paper.

For arithmetic hyperbolic surfaces Selberg proved a faster growth of the remainder: $R(\lambda)=\Omega\left(\frac{\sqrt{\lambda}}{\log \lambda}\right)$ (see [12]). An improvement of this bound for the modular surface $S L(2, \mathbf{Z}) / \mathbf{H}$ was recently obtained in [17; see also 18 for some related estimates. It is conjectured in [24] that on any surface of constant negative curvature $R(\lambda)=O\left(\lambda^{\frac{1}{2}+\epsilon}\right)$ for any $\epsilon>0$. However, the best known upper bound is $R(\lambda)=O(\lambda / \log \lambda)([2])$.

On a generic negatively curved surface it is believed that $R(\lambda)=O\left(\lambda^{\epsilon}\right)$ for any $\epsilon>0$. Such an estimate looks plausible in view of the results on spectral fluctuations, e.g. in [3], [5] and [1]. We note the difference between the predicted upper bound for the global error term, and the lower bound of Theorem 2.4 for the local remainder.

\section{SOME IDEAS OF THE PROOFS}

Let us focus on Theorem 2.6 and indicate some key ideas that are common for the proofs of all the main results (for complete proofs see [15]).

Consider the even part of the wave kernel $e(t, x, y)$ on $X$. It satisfies

$$
e(t, x, y)=\sum_{i=0}^{\infty} \cos \left(\sqrt{\lambda_{i}} t\right) \phi_{i}(x) \phi_{i}(y) .
$$

Take a smooth function $\psi \in C_{0}^{\infty}(\mathbf{R})$ such that supp $\psi \subseteq[-1,1]$, it is even and monotone decreasing on $[0,1], \psi \geq 0, \psi(0)=1$. Fix two positive parameters $\lambda, T$ and consider the function (cf. [16])

$$
k_{\lambda, T}(x, y)=\int_{-\infty}^{\infty} \frac{\psi(t / T)}{T} \cos (\lambda t) e(t, x, y) d t .
$$

We have

Lemma 3.3. If $N_{x, y}(\lambda)=o\left(\lambda^{a}(\log \lambda)^{b}\right), a, b>0$, then $k_{\lambda, T}(x, y)=o\left(\lambda^{a}(\log \lambda)^{b}\right)$.

Lemma 3.3 is used to prove Theorem 2.6 by contradiction. Assuming the contrary, we use a pretrace formula (3.6) to show that $k_{\lambda, T}(x, y)$ is large.

Consider the fundamental solution $E(t, x, y)$ of the wave equation on the universal cover $M$ of $X$. Then given $x, y \in X$, we have

$$
e(t, x, y)=\sum_{\omega \in \Gamma} E(t, x, \omega y)
$$

where the sum is taken over $\Gamma=\pi_{1}(X)$. Let $K_{\lambda, T}(x, y)$ be the analogue of $k_{\lambda, T}(x, y)$ corresponding to the wave kernel $E(t, x, y)$ on $M$ :

$$
K_{\lambda, T}(x, y)=\int_{-\infty}^{\infty} \frac{\psi(t / T)}{T} \cos (\lambda t) E(t, x, y) d t .
$$

Then

$$
k_{\lambda, T}(x, y)=\sum_{\omega \in \Gamma} K_{\lambda, T}(x, \omega y) .
$$


We use the parametrix for $E(t, x, y)$ (see [2], 32]):

$$
E(t, x, y)=\frac{1}{\pi^{\frac{n-1}{2}}}|t| \sum_{j=0}^{\infty} u_{j}(x, y) \frac{\left(r^{2}-t^{2}\right)_{-}^{j-\frac{n-3}{2}-2}}{4^{j} \Gamma\left(j-\frac{n-3}{2}-1\right)} \quad \bmod C^{\infty}
$$

where $r=d(x, y)$. The expression (3.7) is understood in the sense of generalized functions [11. The coefficients $u_{j}(x, y)$ are the solutions of the transport equations along the geodesic joining $x$ and $y$ (see [2]). We recall that $u_{j}(x, x)=a_{j}(x)$ as defined in (1.5).

Asymptotic analysis of the leading terms in (3.7) (cf. 2], 9]) yields

Proposition 3.8. The integral $K_{\lambda, T}(x, y)$ defined by (3.5) satisfies, for any $x \neq$ $y \in M$ as $\lambda \rightarrow \infty$,

$$
K_{\lambda, T}(x, y)=\frac{Q_{0} \lambda^{\frac{n-1}{2}} \psi(r / T)}{T \sqrt{g(x, y) r^{n-1}}} \sin \left(\lambda r+\phi_{n}\right)+O\left(\lambda^{\frac{n-3}{2}}\right) .
$$

Here $g=\sqrt{\operatorname{det} g_{i j}}$ in geodesic normal coordinates, $\phi_{n}=\frac{\pi}{4}(3-(n \bmod 8)), Q_{0}$ is a non-zero constant.

It follows from (3.6), (3.9), and the rate of growth for the number of lattice points on a negatively curved manifold, that

$$
k_{\lambda, T}(x, y)=\sum_{\omega: r_{\omega} \leq T} \frac{Q_{0} \lambda^{\frac{n-1}{2}} \psi\left(\frac{r_{\omega}}{T}\right)}{T \sqrt{g(x, \omega y) r_{\omega}^{n-1}}} \sin \left(\lambda r_{\omega}+\phi_{n}\right)+O\left(\lambda^{\frac{n-3}{2}}\right) \exp (O(T)) .
$$

Let us now bound from below the following sum:

$$
S_{x, y}(T)=\sum_{r_{\omega} \leq T, \omega \in \Gamma} \frac{1}{\sqrt{g(x, \omega y) r_{\omega}^{n-1}}},
$$

where $y \notin \Gamma x, r_{\omega}=d(x, \omega y)$.

The sum (3.11) can be estimated by a sum over closed geodesics on $X$. This is possible due to the fact that near a geodesic segment $[x, \omega y]$ there exists a closed geodesic of comparable length (a similar idea was applied in [4]). Reduction to closed geodesics requires some careful analysis of the behavior of the geodesic flow.

We apply thermodynamic formalism to bound the sum over closed geodesics from below. Namely, using the results of [19, 20], we get

Theorem 3.12. There exists a constant $C_{0}>0$ such that

$$
S_{x, y}(T) \geq C_{0} e^{P\left(-\frac{\mathcal{H}}{2}\right) \cdot T}
$$

as $T \rightarrow \infty$, where $P$ is the topological pressure (2.3) and $\mathcal{H}$ is the Sinai-RuelleBowen potential (2.2).

One can show that $P\left(-\frac{\mathcal{H}}{2}\right) \geq \frac{(n-1) K_{2}}{2}$, hence $S_{x, y}(T)$ grows exponentially in T.

To get a contradiction with Lemma 3.3. we consider the expression $k_{\lambda, T}(x, y)$, and let $T$ grow with $\lambda$. We then use Dirichlet box principle (cf. [22, 25]) to choose $\lambda$ so that $\lambda r_{\omega}$ be very close to an integer multiple of $2 \pi$ for all $\omega$ with $r_{\omega} \leq T$. In order to make the Dirichlet box principle work we have to choose $T$ of the size $\frac{1}{h} \ln \ln \lambda$. This together with (3.13) explains the logarithmic factor in Theorem 2.6.

Assume now that $n \not \equiv 3(\bmod 4)$. Then all the expressions $\sin \left(\lambda r_{\omega}+\phi_{n}\right)$ in (3.10) are of the same sign and bounded away from zero by a constant. Combining this 
with the estimate (3.13), we obtain a lower bound for the right-hand side of (3.10), which leads to the desired contradiction with Lemma 3.3.

When $n \equiv 3(\bmod 4), \phi_{n}=0$ in (3.9) and the Dirichlet box principle cannot be used directly. We modify the argument in order to bound from below all the expressions $\sin \left(\lambda r_{\omega}\right)$ in (3.10) for $T / A \leq r_{\omega} \leq T$, where $A$ is a suitably chosen constant. This proves Theorem 2.6 in all dimensions.

\section{ACKNOWLEDGEMENTS}

The authors are deeply grateful to D. Dolgopyat for explaining them hyperbolic dynamics leading to Theorem 3.12 We would like to thank V. Ivrii, M. Jakobson, V. Jaksic, Y. Kannai, J. Marklof, Ya. Pesin, M. Pollicott, L. Polterovich, A. Reznikov, P. Sarnak, R. Sharp, A. Shnirelman, M. Shubin, J. Toth and D. Wise for useful discussions. We would also like to thank the anonymous referee for helpful

remarks. This paper was completed while the first author visited IHES, and its hospitality is greatly appreciated.

\section{REFERENCES}

[1] R. Aurich, J. Bolte, F. Steiner. Universal signatures of quantum chaos. Phys. Rev. Lett. 73 (1994), no. 10, 1356-1359.

[2] P. Berard. On the wave equation on a compact riemannian manifold without conjugate points. Math. Z. 155 (1977), 249-276. MR0455055 (56:13295)

[3] M. V. Berry. Semiclassical theory of spectral rigidity. Proc. R. Soc. Lond. A 400 (1985), 229251. MR0805089 (87i:81053)

[4] E. Bogomolny. Smoothed wavefunctions of chaotic quantum systems. Physica D 31 (1988), 169-189. MR0955627 (89h:81062)

[5] E. Bogomolny, C. Schmit. Semiclassical computations of energy levels. Nonlinearity 6 (1993), 523-547. MR 1231772 (94i:81027)

[6] R. Bowen. Equilibrium states and the ergodic theory of Anosov diffeomorphisms. Lecture Notes in Math. 470. Springer, 1975. MR0442989 (56:1364)

[7] R. Bowen and D. Ruelle. The ergodic theory of Axiom A flows. Invent. Math. 29 (1975), no. 3, 181-202. MR0380889 (52:1786)

[8] Y. Colin de Verdière. Spectre conjoint d'opérateurs pseudo-différentiels qui commutent. II. Le cas intégrable. Math. Z. 171 (1980), no. 1, 51-73. MR0566483 (81i:58046)

[9] H. Donnelly. On the wave equation asymptotics of a compact negatively curved surface. Invent. Math. 45 (1978), 115-137. MR0471001 (57:10744)

[10] J. Duistermaat and V. Guillemin. The spectrum of positive elliptic operators and periodic bicharacteristics. Inventiones Math. 29 (1975), 39-75. MR0405514(53:9307)

[11] I. M. Gelfand and G. E. Shilov. Generalized functions, vol. 1, Academic Press, New York, 1964. MR0435831(55:8786a)

[12] D. Hejhal. The Selberg trace formula for PSL(2, R), Vol. I. Lecture Notes in Math. 548, Springer, 1976. MR0439755 (55:12641)

[13] L. Hörmander. The spectral function of an elliptic operator. Acta Math. 121 (1968), 193-218. MR0609014 (58:29418)

[14] V. Ivrii. Precise spectral asymptotics for elliptic operators. Lecture Notes in Math. 1100, Springer, 1984. MR0771297 (86h:58139)

[15] D. Jakobson, I. Polterovich. Estimates from below for the spectral function and for the remainder in local Weyl's law, math.SP/0505400.

[16] A. Karnaukh. Spectral count on compact negatively curved surfaces. Ph.D. thesis under the supervision of P. Sarnak, Princeton University (1996), 1-48.

[17] X. Li and P. Sarnak. Number variance for $S L(2, \mathbf{Z}) / \mathbf{H}$. Preprint 2004.

[18] W. Luo and P. Sarnak Number variance for arithmetic hyperbolic surfaces. Comm. Math. Phys. 161 (1994), 419-432. MR1266491 (95k:11076) 
[19] W. Parry. Equilibrium states and weighted uniform distribution of closed orbits. Dynamical Systems (College Park, MD 1986-87), Lecture Notes in Math. 1342, 617-625. Springer, 1988. MR0970574 (90a:58095)

[20] W. Parry and M. Pollicott. Zeta functions and closed orbit structure for hyperbolic systems. Astérisque, 187-188 (1990), 1-256. MR:1085356 (92f:58141)

[21] Y. Petridis and J. Toth. The remainder in Weyl's law for Heisenberg manifolds. J. Diff. Geom. 60 (2002), no. 3, 455-483. MR.1950173 (2004c:58054)

[22] R. Phillips and Z. Rudnick. The circle problem in the hyperbolic plane. J. Funct. Anal. 121 (1994), no. 1, 78-116. MR.1270589 (95d:11135)

[23] B. Randol. The Riemann hypothesis for Selberg's zeta-function and the asymptotic behavior of eigenvalues of the Laplace operator. Trans. Amer. Math. Soc. 236 (1978), 209-223. MR.0472728(57:12420)

[24] B. Randol. A Dirichlet series of eigenvalue type with applications to asymptotic estimates. Bull. London Math. Soc. 13 (1981), 309-315. MR0620043 (82j:10082)

[25] M. Rubinstein and P. Sarnak. Chebyshev's bias. Experiment. Math. 3 (1994), no. 3, 173-197. MR1329368 (96d:11099)

[26] Y. Safarov and D. Vassiliev. The asymptotic distribution of eigenvalues of partial differential operators. Translations of Mathematical Monographs, 155. AMS, 1997. MR1414899 (97h:58163)

[27] Ya. Sinai. Gibbs measures in ergodic theory. Uspehi Mat. Nauk 27 (1972), 4(166), 21-64. English transl., Russian Math. Surveys 27 (1972), 21-69. MR0399421 (53:3265)

[28] M. Shubin. Pseudodifferential operators and spectral theory, Springer-Verlag, 1987. MR0883081 (88c:47105)

[29] C. Sogge and S. Zelditch. Riemannian manifolds with maximal eigenfunction growth. Duke Math. J. 114 (2002), no. 3, 387-437. MR.1924569 (2004b:58053)

[30] K.-M. Tsang. Counting lattice points in the sphere. Bull. London Math. Soc. 32 (2000), no. 6, 679-688. MR 1781580 (2001h:11129)

[31] A. Volovoy. The Hamilton flow conditions associated with Weyl's conjecture, Ann. Global Anal. Geom. 8, No. 2 (1990), 127-136. MR.1088506 (91m:58157)

[32] S. Zelditch, Lectures on wave invariants, in: Spectral theory and geometry, edited by B. Davies and Y. Safarov, LMS lecture note series 273, Cambridge University Press, 1999. MR 1736870(2001a:58044)

Department of Mathematics and Statistics, McGill University, 805 Sherbrooke St. West, Montréal QC H3A 2K6, Canada

E-mail address: jakobson@math.mcgill.ca

Département de mathématiques et de statistique, Université de Montréal CP 6128 Succ. Centre-Ville, Montréal QC H3C 3J7, Canada

E-mail address: iossif@dms.umontreal.ca 